\title{
Location, Location, Location: An Analysis of Profitability of Position in Online Advertising Markets
}

\author{
Ashish Agarwal \\ Tepper School of Business, Carnegie Mellon University, ashish1agarwal@cmu.edu \\ Kartik Hosanagar \\ The Wharton School, University of Pennsylvania, kartikh@wharton.upenn.edu \\ Michael D. Smith \\ Heinz School of Public Policy and Management, Carnegie Mellon University, \\ mds@cmu.edu
}

This Version: November 2008

Acknowledgements: The authors thank Professor Peter Fader, Christophe Van den Bulte, seminar participants at the 2007 Workshop on Information Systems and Economics (WISE) and the 2008 Marketing Science conference for valuable comments on this research. Smith acknowledges the National Science Foundation for generous financial support provided through CAREER award IIS-0118767. 


\begin{abstract}
$\underline{\text { ABSTRACT }}$
Sponsored search accounts for $40 \%$ of the total online advertising market. These ads appear as ordered lists along with the regular search results in search engine results pages. The conventional wisdom in the industry is that the top position is the most desirable position for advertisers. This has led to intense competition among advertisers to secure the top positions in the results pages.

We evaluate the impact of ad placement on revenues and profits generated from sponsored search using data for several hundred keywords from the ad campaign of an online retailer. Using a hierarchical Bayesian model, we measure the impact of ad placement on both click-through rate and conversion rate for these keywords. We find that while click through rate decreases with position, conversion rate first increases and then decreases with position for longer keywords. The net effect is that, contrary to conventional wisdom, the topmost position in sponsored search advertisements is not necessarily the revenue- or profit-maximizing position. Our results inform the advertising strategies of firms participating in sponsored search auctions and provide insight into consumer behavior in these environments. Specifically, they help correct a significant misunderstanding among advertisers regarding the value of the top position. Further, they reveal potential inefficiencies in present auction mechanisms used by the search engines.
\end{abstract}

Keywords: Sponsored search, ad placement, hierarchical Bayesian estimation, online advertising, online auctions, search engine marketing 
Internet advertising spend is currently growing faster than any other form of advertising and is expected to grow from $\$ 16.4$ billion in 2006 to $\$ 36.5$ billion in 2011 (eMarketer). $40 \%$ of this ad spend occurs on sponsored search, where advertisers pay to appear alongside the regular search results of a search engine. Most search engines, including Google, Yahoo, and MSN, use auctions to sell their ad space inventory. In these auctions, advertisers submit bids on specific keywords based on their willingness to pay for a click from a consumer searching on that (or a closely related) keyword. Search engines use a combination of the submitted bid and past click performance to rank order the ads. Sponsored search is unique relative to offline advertising and other forms of online advertising because it is presumed to occur close to a user's purchase decision and is matched based on the user's stated information need (Hosanagar and Cherapanov 2008). As a result, advertisers are spending a greater share of their advertising budgets on search engine marketing and are often engaged in intense bidding wars to win the top slots in the list of sponsored results (Target Marketing 2006; Wall Street Journal 2007).

The rationale behind these bidding wars for top positions is that Click through Rates (CTR) typically decrease exponentially with ad position and thus the top few positions receive the majority of clicks. This thinking is well summarized by the following quote posted on a search engine forum: ${ }^{1}$

"I believe that people who think it's better to be anything other than \#1 are just fooling themselves... The fact lies that you'll get 3 1/2 times more traffic being \#1 as opposed to \#2, and the numbers keep sliding from there."

Based on this conventional wisdom, most advertisers aggressively seek the topmost positions in their bidding, and Search Engine Marketing (SEM) firms that offer bidding services to

\footnotetext{
${ }^{1}$ The quote is based on analysis of click-through rates observed in the top ten algorithmic search positions in AOL's dataset. However, similar thinking is prevalent for sponsored search as well.
} 
advertisers often provide guarantees to clients of securing the top positions. However, there have been few formal studies of the impact of ad position on click through rates, conversion rates (i.e. the likelihood that a consumer will buy a product) and advertising costs. ${ }^{2}$ Thus, the net impact of ad position on overall revenues and profits is not well understood.

In this paper we address this question by empirically analyzing how ad position in sponsored search impacts an advertiser's revenues and overall profits. We use a unique panel dataset from a Search Engine Marketing (SEM) firm that catalogs daily clicks, conversions, and cost data for multiple keywords sponsored by one of its clients. One of the challenges with sponsored search data is that clicks and conversions are sparse. In order to address this, we use a hierarchical Bayesian model to analyze the click and conversion probabilities in this environment while accounting for heterogeneity across keywords. Our findings suggest that, contrary to conventional wisdom, the topmost positions for keywords in our dataset are associated with lower revenues relative to lower (and less expensive) positions. Our results confirm that ad clickthrough rate decreases with position. However, we find that the conversion rate and revenue initially increase and then decrease with ad position for longer keyphrases. For shorter keyphrases, the revenue decreases with position. However, the costs are much higher in the top positions resulting in higher profits at lower position.

Our paper makes two main contributions. First, our paper provides key managerial insights for advertisers. A common assumption in the industry is that the value of a click from a sponsored search campaign is independent of the position of the advertisement. Our results indicate this is not true, and moreover that a click from an ad at the top position may have a

\footnotetext{
${ }^{2}$ The term "conversion" is more commonly used than "purchase" because the definition of successful customer acquisition varies by firm. For example, for some firms, such as a free email service provider, the act of creating an account is referred to as a conversion, and is more meaningful metric of success than a purchase. In our analysis, conversion rate is the rate at which consumers buy after seeing an advertisement.
} 
lower expected revenue as compared to a click from the same ad placed lower in the list of advertisements. As a result, we find that the top ad positions do not maximize advertiser revenues or profits in case of popular keywords, and thus advertisers should revisit the assumptions driving current bidding wars for the top ad positions.

Second, our results highlight potential inefficiencies in the rules commonly used in sponsored search auctions, and suggest the need for further investigation of these pricing mechanisms. Previous studies have evaluated sponsored search auction mechanisms using theoretical constructs (Chen and He, 2006; Edelman, Ostrovsky \& Schwarz, 2007) and empirical analysis of the advertiser's bidding behavior (Edelman \& Ostrovsky, 2007). However, actual consumer behavior has rarely been studied. We use consumer choice models to evaluate revenue and profit differences across the different positions assigned in the auction. If advertisers with the best combination of bid and CTR are assigned the top position, but lower positions generate higher revenues, then current auction rules may well be doing these advertisers a disservice. Our results suggest that using CTR and other click-oriented measures alone to determine ad ordering may not be sufficient, and that more conversion-oriented metrics for ad ordering and pricing can help increase the efficiency of the market.

It is important to note that our study is conducted from the perspective of transactional revenue and profit. We do not consider non-transactional benefits, such as increased product or brand awareness in our analysis. However, while not fully discounting such possibilities, we note that surveys of online advertisers indicate that $99 \%$ of advertisers use search engine advertising to drive direct transactional benefits such as immediate sales or profits (Kitts et al. 2005). Furthermore, the prevailing wisdom in the industry does not reflect an appreciation of the variability of conversions by ad position. Thus, while we believe our results are applicable to a 
wide range of industries, our results will be less applicable in industries where the goal of the online advertisement is primarily to increase exposure, awareness, or branding.

We organize the rest of the article as follows. We begin with an explanation of the sponsored search market and describe prior academic work in this area. Next, we develop a model to measure the performance of sponsored search ads. Then we describe the data and the estimation approach. Thereafter, we discuss the empirical results. Finally we conclude the study, discuss limitations, and areas for future research.

\section{BACKGROUND \& LITERATURE REVIEW}

\section{Sponsored Search}

When a consumer enters a search query, for example "Digital Camera," the search engine displays algorithmic (i.e. regular), and sponsored search results as shown in Figure 1. The algorithmic results are determined based on their relevance to the query. The sponsored results are ranked based on continuous real-time auctions run by the search engines. Advertisers bid on sponsored search keywords of relevance to them. Upon receiving a query, the search engine identifies the advertisers bidding on closely related keywords, and uses data on bids and past click performance of ads to rank order the ads that appear in the list of sponsored results.

An advertiser pays the search engine only when the consumer clicks on the advertiser's ad. The cost per click (CPC) is determined using a generalized second price auction mechanism; i.e. whenever a user clicks on an ad in position $k$, the advertiser pays an amount equal to the minimum bid needed to secure that position (Lahaie et al. 2007). After clicking on the ad, the consumer is redirected to the advertiser's website, and then chooses whether to purchase a product or register for a service (which we define as conversions). 


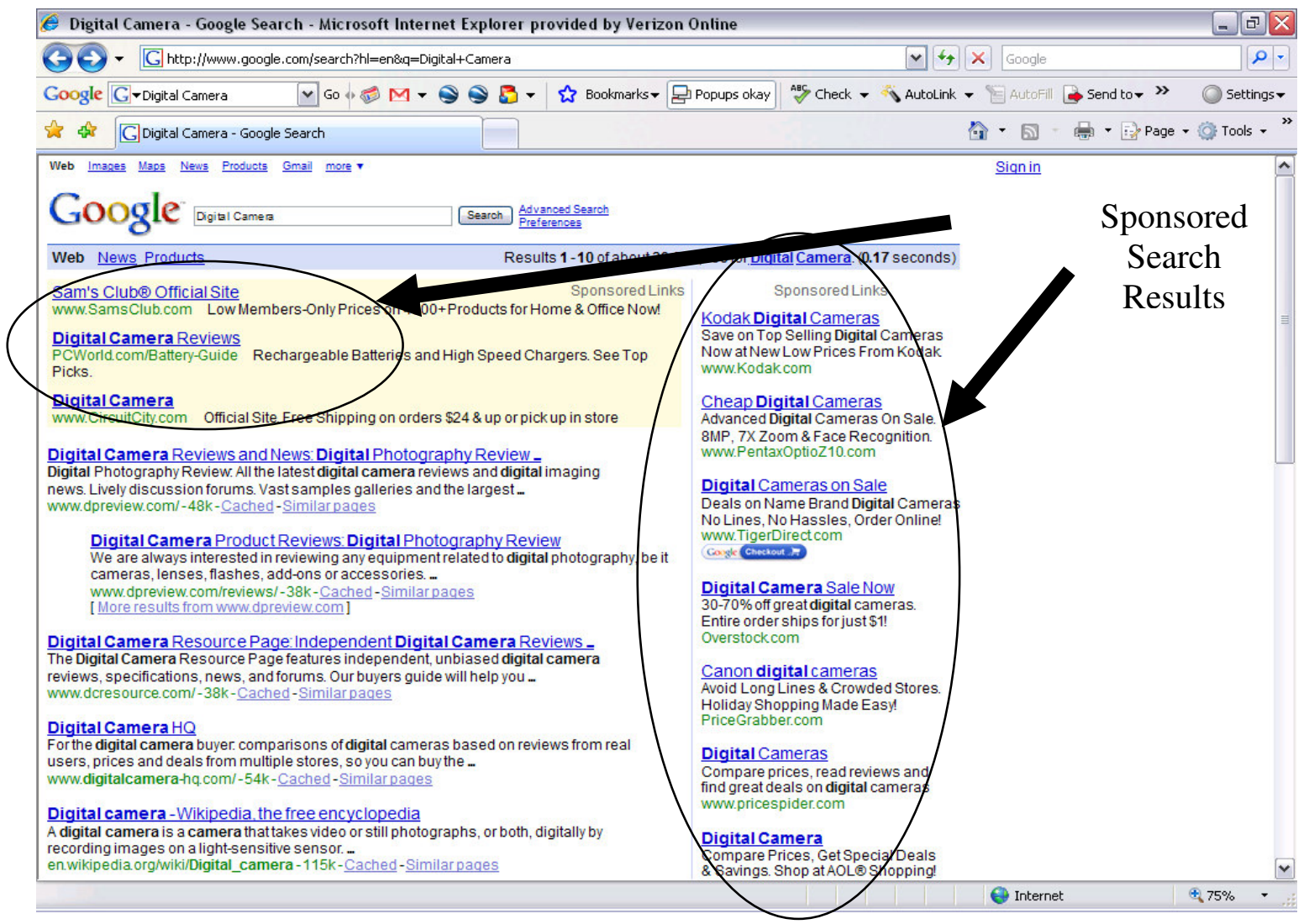

Figure 1: Search Results

The search engines provide daily reports to advertisers on the status of their campaigns. These reports provide statistics on the number of impressions and clicks, and report the average position for each keyword in the advertiser's portfolio. The continuous nature of the auction allows an advertiser to change the portfolio of keywords as well as the bids, ad copies, and landing pages for each keyword in real-time. The bid submitted by the advertiser implicitly determines the target position for the ad. These decisions ultimately drive the advertiser's Return on Ad Spend (RoAS), a key metric used to evaluate return on investments in advertising. In our study, we focus on the impact of the advertisement's position in the list of sponsored search results on revenues and profitability for a given set of keywords. The advertisements and landing pages related to these keywords do not change over time for the advertiser under consideration. 


\section{Prior Work}

The literature most relevant to our study includes past research on consumers' online search behavior, with a special emphasis on the impact of message order on consumer choice and the research focused on advertisers' performance in sponsored search markets.

Consumers' online search behavior: An important consideration in evaluating the performance of the sponsored search advertisements is the consumer response to the ad position, and the depth of consumer search. Prior work in traditional media has demonstrated that message ordering influences ad persuasion (Rhodes et al. 1973, Brunel and Nelson, 2003). It is thus likely that ordering and position strongly influence the attention paid to a marketing message online. Consistent with that, Hoque and Lohse (1999) find that consumers are more likely to choose advertisements near the beginning of an online directory as compared to paper directories. Prior studies have also shown that the depth of consumer search on the Internet is low. For example, Johnson et al. (2004) found that consumers searched fewer than two stores during a typical search session. Similarly, Brynjolfsson, Dick, and Smith (2006) find that only $9 \%$ of users of a shopbot select offers beyond the first page. Although recent studies suggest some increase in the number of stores evaluated (Zhang et al. 2007), search depth still remains limited. Due to cognitive costs associated with evaluating alternatives, consumers often focus on a smaller set of results (Montgomery et al. 2004). Consumers may gain very little by adding additional items into their consideration set (Hauser and Wernerfelt, 1990). Beardan, Rapport and Murphy (2006) find that in environments with sequential evaluation of choices and rank dependent payoffs, search is terminated too early. Additionally, the quality of the earlier choices is overestimated. A natural hypothesis in the case of sponsored search is that consumers focus on the top positions in the list 
of results. Feng et al. (2007) find evidence of an exponential decrease in the number of clicks for an ad with its rank, and attribute this to decay in user attention as one proceeds down a list.

Search behavior is also dictated by the consumer's purchase intent. Consumer search can be goal directed or exploratory (Janiszewski 1998). Online consumers include both buying consumers and information seekers (Moe 2003, Moe \& Fader, 2004; Montgomery, Li, Srinivasan, \& Lietchy, 2004). Consumers with high purchase intent tend to be very focused in their search, targeting a few products and categories versus consumers with low purchase intent, who have broad search patterns targeting a higher variety of products (Moe 2003). A similar pattern can be expected in sponsored search i.e. consumers may be heterogeneous in terms of their purchase intent and resulting search behavior.

Search engines can also be viewed as tools that aid consumer decision-making. Haubl and Trifts (2000) find that the use of decision aids reduces the size of consumers' consideration set but improves the quality of their consideration sets and the quality of purchase decisions in the online shopping environment. However, the sponsored search advertisements reveal only limited information, and the products can be evaluated only after clicking the ads and visiting the advertiser's website. Previous studies show that consumers tend to de-emphasize the prescreening information in their search process (Diel, Kornish and Lynch, 2003; Chakravarti et. al 2006). This suggests that the criteria used for selecting an ad to click may not have an effect on the final conversion as compared to the information obtained after visiting the associated website. Thus, the overall impact of rank ordering of sponsored search ads on the purchase decisions is not clear.

Sponsored search markets: Existing work in sponsored search has focused on advertiser strategy, auction design, and consumer behavior. A consequence of the sequential evaluation of 
ads is that the top positions may become more valuable for advertisers. Thus advertisers aggressively vie for top positions. Ganchev et al. (2007) provide empirical evidence of exponential decay of bids with position. Arbatskaya (2007) provides a theoretical explanation for the decrease in equilibrium prices and profits in the order of search for homogenous goods. These results justify the decay in the bids with position based on the assumption that the revenue will indeed decay with position. However, it is not clear whether this will be the case when the consumers are uncertain about the quality of the underlying products. Due to the uncertainty, consumers may evaluate more than one advertisement. In that case consumers have to compare products across multiple advertisements and may have to revisit previously evaluated products incurring additional browsing costs.

There has also been recent work on optimal bidding strategies of advertisers (Hosanagar and Cherapanov 2008; Borgs et al. 2007; Feldman et al. 2007; Cary et al. 2007). These papers present optimization models to compute the bids for all keywords in an advertiser's portfolio in order to maximize advertiser profits subject to a budget constraint. The models capture the notion that a high bid ensures a top position and therefore generates a large volume of clicks, but correspondingly incurs a high cost per click. However, none of these papers have explicitly studied the impact of ad position on advertiser revenues and profitability. These papers have mainly focused on optimal budget allocation among a large set of heterogeneous keywords. Furthermore, all these papers assume that every click for a keyword is worth the same regardless of the ad's position. As we find in our dataset, this ignores a crucial dependence between ad position and conversion rates.

Another area of focus has been the evaluation of the sponsored search auction design and its impact on the advertisers' bidding strategies. Edelman et al. (2005) and Varian (2006) compute 
the equilibria of the generalized second price sponsored search auction and demonstrate that the auction, unlike the Vickrey-Clarke-Groves (VCG) mechanism, is not incentive compatible. Thus, advertisers will bid strategically in these auctions. Edelman and Ostrovsky (2007) examine data on paid search auctions and find evidence of strategic bidder behavior. Feng et al. (2007) and Weber and Zhang (2007) compare the performance of various ad ranking mechanisms. They find that a yield-optimized auction, with ranking based on a combination of the submitted bid and ad relevance, provides the highest revenue to the search engine. Some studies have also focused on the quality ordering of advertisements. Chen and He (2006) show that when advertisers are differentiated, they bid according to product relevance. The corresponding paid placement by the search engine results in efficient search by the consumers and increases the social surplus. In contrast, Animesh et al. (2006) empirically show that auctions that do not account for ad relevance are more likely to display lower quality ads in top positions, especially for experience goods and credence goods. Similarly, Esteban et al. (2006) find that customer-directed variablecost advertising can lead to quality distortions and lower social welfare. Dellarocas and Viswanathan (2007) also study quality signaling and revenue implications of sponsored search advertising. They focus on single slots and do not consider the quality variation across multiple positions. An implicit assumption in all these papers is that the higher rank is more valuable for the advertisers.

Finally, recent empirical studies have modeled consumer choice in sponsored search. Rutz and Bucklin (2007) show that keyword characteristics are important for determining conversion performance. Using an aggregated dataset, Ghose and Yang (2007) also model clicks and conversions and show that firms use data on past performance to improve their future performance. They also run policy simulations to determine the optimal bid prices. Goldfarb and 
Tucker (2007) show that when there is significant match difficulty between consumers and advertisers, advertisers are likely to bid higher. Misra and Pinker (2006) find that ad position has a higher impact on click through rate at Google as compared to Yahoo. However, none of these studies focus on the bid efficiency of position or how ad position influences the profitability of sponsored search advertising.

Thus, the prior literature reveals two themes. First, the literature on consumer search behavior suggests that ad position is likely to influence consumer response. Second, recent work in sponsored search confirms that clicks decay with ad position, but indicates that so do costs. Thus, the net effect on profits is not easily predictable. Moreover, the impact of position on purchase likelihood or conversion rate is unknown. Thus, the net impact of position on both revenues and profitability is an open and managerially significant research question.

\section{MODEL}

Consider an advertiser placing bids for a keyword in order to ensure its ads are visible in the list of sponsored results for a query related to that keyword. Our interest is in determining the impact of the ad's position, $n$, on the advertiser's expected profit $\pi(n)$, where $\pi(n)$ is given by

$\pi(\mathrm{n})=\mathrm{I} \times(\mathrm{CVI}(\mathrm{n}) \times \mathrm{RPC}-\mathrm{CTR}(\mathrm{n}) \times \mathrm{CPC}(\mathrm{n}))$

and where I is the expected number of ad impressions. CTR(n) is the click through rate, or the fraction of ad impressions that generate clicks (given n). CVI(n) is the conversion rate per impression, or the fraction of impressions that generate conversions (e.g. purchases) ${ }^{3}$. RPC is the average revenue per conversion. Finally, $\mathrm{CPC}(\mathrm{n})$ is the average cost per click charged to an

\footnotetext{
${ }^{3}$ Alternatively, conversions can be modeled as a two step process: consumers first click an ad and then buy a product. We have also estimated our model using this two step approach and get the same qualitative results.
} 
advertiser that is assigned position $n$. We assume that the number of impressions is independent of the position of the advertisements.

A common challenge encountered in this environment is that many keywords do not get any clicks for several days. Thus the clicks and conversions data for individual keywords are sparse. Further, there is considerable heterogeneity across the keywords as the competing advertisers and the corresponding ads may be different for different keywords. In order to overcome this problem of data sparseness and keyword heterogeneity, we use a Hierarchical Bayesian model (HB Model), to pool observations across keywords sharing similar characteristics. This provides a flexible random component specification that allows us to incorporate both observable and unobservable keyword-specific heterogeneity. Hierarchical models are commonly used to draw inferences on individual level characteristics from sparse datasets (Rossi \& Allenby, 2003), and HB models have recently been applied to study sponsored search data (Rutz and Bucklin, 2007; Ghose and Yang, 2007).

A common concern for demand specification in brand choice models is the endogeneity of some of the independent variables such as price (Villas-Boas and Winer, 1999). This endogeneity can arise due to both consumers and advertisers. Consumers may demonstrate strategic behavior when they engage in repeat purchases. A key difference, however, in our setting is that consumers are not buying products in our sample very often. Additionally, consumers can use different sets of keywords to meet their search goals. The information processing costs are high in an online setting and consumers are unlikely to remember the relative positions of the ads even if they repeat the search for a keyword. As a consequence, consumers are unlikely to demonstrate any strategic behavior in terms of position-based choices. Further any demand shocks are unlikely to change the relative ordering of the advertisements. 
Another possible source of endogeneity is that the advertiser has selected its bid based on the click and conversion performance of the keywords. Further search engines take into account the bids of all the advertisers along with their past performance to determine their position in the sponsored search slots. To address this concern, we use a recursive system of equations to account for the bid and the corresponding ad positions. To further address potential endogeneity due to advertiser behavior, we conducted separate experiments wherein the bids for ten keywords were randomly selected within a lower and upper bound. Although the coefficients estimated from the random bids vary, our results are directionally consistent in this smaller sample of keywords. These results are described in the Appendix A2. Because the scope of the experiment was limited to 10 keywords, our main analysis and results are based on the full sample.

\section{Conversion Rate per Impression (CVI)}

An important consideration for purchase is the relevance, or quality of the product associated with the advertisement. Consumers may form an expectation that the advertisements are arranged in a decreasing order of relevance or quality. This is potentially more likely in the sponsored search environment, where the consumers are used to the relevance-based ordering of non-sponsored search results. Conversion rates may reflect these differences in relevance. Alternatively, they may reflect differences in the types of customers that click on ads at top positions relative to those that click lower ranked ads. Consumer choice to buy a product by clicking an ad and then purchasing can be modeled using a latent utility model for conversion. For a keyword $\mathrm{k}$ at time $\mathrm{t}$, this latent utility can be expressed as

$$
\begin{aligned}
& \mathrm{U}_{\mathrm{kt}}^{\mathrm{CVI}}=\mathrm{Y}_{\mathrm{kt}}^{\mathrm{CVI}}+\varepsilon_{\mathrm{kt}}^{\beta} \\
& \mathrm{Y}_{\mathrm{kt}}^{\mathrm{CVI}}=\beta^{\mathrm{k}} \mathrm{X}_{\mathrm{kt}}^{\beta}
\end{aligned}
$$


$\beta^{\mathrm{k}}=\Delta^{\beta} \mathrm{z}_{\mathrm{k}}+\mathrm{u}_{\mathrm{k}}^{\beta} \quad \mathrm{u}_{\mathrm{k}}^{\beta} \sim \mathrm{N}\left(0, \mathrm{~V}^{\beta}\right)$

where the covariates are as follows:

$\operatorname{pos}_{\mathrm{kt}}$ is the ad position for a particular advertiser for keyword $\mathrm{k}$ at time $\mathrm{t}$,

$\operatorname{pos}_{\mathrm{kt}}{ }^{2}$ is the square term for the ad position. It allows us to capture any non-monotonic patterns, $\delta_{\mathrm{kt}}^{\mathrm{m}}$ is an indicator function to control for the month. This is used to capture seasonality in the selection of an ad and the underlying products. For example, ads related to 'swimwear' are most popular in late spring and summer,

and a constant term.

$\mathrm{z}_{\mathrm{k}}$ represents keyword specific characteristics; which in our case is the number of words in the keyphrase, labeled "length". For example, keyword 'pants' has a length of one, whereas the keyword 'brown casual pants' has a length of three. A high value of "length" generally indicates that the keyword is more specific. ${ }^{4}$ Keywords can be further characterized in terms of the product category, product popularity, and other features. However, studying the effect of these characteristics is not the focus of this research paper. We use a constant term to capture the net effect of these other characteristics.

$\Delta^{\beta}$ is a matrix which captures the relationship between the keyword characteristics and the mean values of the coefficients.

$u_{k}^{\beta}$ represents the unobservable heterogeneity for each keyword, which we assume is normally distributed with a mean 0 and covariance matrix $\mathrm{V}^{\beta}$

We assume that $\varepsilon_{\mathrm{kt}}^{\beta}$ are i.i.d with an extreme value distribution. Correspondingly, we use a logit model to represent the conversion probability for a keyword $\mathrm{k}$ at time $\mathrm{t}$ as follows

\footnotetext{
${ }^{4}$ The specificity of a keyword can also be expressed in terms of semantics. For example, the keyword 'pants' is more specific than the keyword 'clothing.' However, we do not account for these semantic differences in the present analysis.
} 
$\Lambda_{\mathrm{kt}}^{\mathrm{CVI}}=\frac{\exp \left(\mathrm{Y}_{\mathrm{kt}}^{\mathrm{CVI}}\right)}{1+\exp \left(\mathrm{Y}_{\mathrm{kt}}^{\mathrm{CVI}}\right)}$

The corresponding likelihood function is

$\mathrm{l}_{\text {Conversion }}=\prod_{\mathrm{t}=1}^{\mathrm{T}} \prod_{\mathrm{k}=1}^{\mathrm{K}}\left(\Lambda_{\mathrm{kt}}^{\mathrm{CVI}}\right)^{\text {Conversions }_{\mathrm{kt}}} \cdot\left(1-\Lambda_{\mathrm{kt}}^{\mathrm{CVI}}\right)^{\text {Impressions }} \mathrm{kt}_{\mathrm{t}}-$ Conversions $_{\mathrm{kt}}$

\section{Click through rate (CTR)}

Consumer choice of selecting an advertisement can be modeled in terms of the latent utility of clicking on an advertisement. For a keyword $k$ at time $t$, this latent utility can be expressed as

$\mathrm{U}_{\mathrm{kt}}^{\mathrm{CTR}}=\mathrm{Y}_{\mathrm{kt}}^{\mathrm{CTR}}+\varepsilon_{\mathrm{kt}}^{\theta}$

$\mathrm{Y}_{\mathrm{kt}}^{\mathrm{CTR}}=\theta^{\mathrm{k}} \mathrm{X}_{\mathrm{kt}}$

$\theta^{\mathrm{k}}=\Delta^{\theta} \mathrm{z}_{\mathrm{k}}+\mathrm{u}_{\mathrm{k}}^{\theta} \quad \mathrm{u}_{\mathrm{k}}^{\theta} \sim \mathrm{N}\left(0, \mathrm{~V}^{\theta}\right)$

Similar to the CVI model, we use linear and square terms for the ad position and have controls for the month and a constant term.

The click probability can be expressed as follows

$$
\Lambda_{\mathrm{k}, \mathrm{t}}^{\mathrm{CTR}}=\frac{\exp \left(\mathrm{Y}_{\mathrm{kt}}^{\mathrm{CTR}}\right)}{1+\exp \left(\mathrm{Y}_{\mathrm{kt}}^{\mathrm{CTR}}\right)}
$$

The resulting likelihood function for clicks for $\mathrm{K}$ keywords over $\mathrm{T}$ periods is

$\mathrm{l}_{\mathrm{Click}}=\prod_{\mathrm{t}=1}^{\mathrm{T}} \prod_{\mathrm{k}=1}^{\mathrm{K}}\left(\Lambda_{\mathrm{kt}}^{\mathrm{CTR}}\right)^{\mathrm{Clicks}_{\mathrm{kt}}} \cdot\left(1-\Lambda_{\mathrm{kt}}^{\mathrm{CTR}}\right)^{\text {Impressions }_{\mathrm{kt}}-\mathrm{Clicks}_{\mathrm{kt}}}$

Similar models have been used by Misra et al. (2006) and Rutz and Bucklin (2006) to model clicks and conversions respectively as a function of ad attributes.

\section{Advertiser's Bid}

The advertiser can potentially change the bid for each keyword in its portfolio on a continuous basis. This decision is typically based on the past performance and future expected performance 
for each keyword. Thus we model the advertiser's bid for each keyword on any day as a function of the past CTR, CVI and profit for different positions. We measure past performance in terms of the performance over the previous seven days. ${ }^{5}$ We use the following reduced form equation to represent the bid for a keyword in the current period ${ }^{6}$

$\ln \left(\operatorname{Bid}_{\mathrm{k}, \mathrm{t}}\right)=\gamma^{\mathrm{k}} \mathrm{X}_{\mathrm{kt}}^{\gamma}+\varepsilon_{\mathrm{kt}}^{\gamma}$

$\gamma^{\mathrm{k}}=\Delta^{\gamma} \mathrm{z}_{\mathrm{k}}+\mathrm{u}_{\mathrm{k}}^{\gamma} \quad \mathrm{u}_{\mathrm{k}}^{\gamma} \sim \mathrm{N}\left(0, \mathrm{~V}^{\gamma}\right)$

where the covariates are as follows:

$\operatorname{pos}_{\mathrm{k}, \mathrm{week}-1}$ is the average position for keyword $\mathrm{k}$ for the past seven days,

$\mathrm{CTR}_{\mathrm{k} \text {,week-1 }}$ is the average CTR for keyword $\mathrm{k}$ for the past seven days,

$\mathrm{CVI}_{\mathrm{k} \text {,week-1 }}$ is the average CVI for keyword $\mathrm{k}$ for the past seven days,

Profit $_{\mathrm{k}, \mathrm{week}-1}$ is the average profit generated for keyword $\mathrm{k}$ in the past seven days,

$\delta_{\mathrm{kt}}^{\mathrm{m}}$ is an indicator function to control for the month,

and a constant term.

\section{Ad Position}

The position of an advertisement for a keyword is based on the product of the current bid and the past performance (also known as quality score) of the advertisement ${ }^{7}$. Search engines typically use past click through rate as a measure of performance as they cannot directly observe conversions and do not typically receive this information from the advertisers. Top positions are more likely to have a higher click through rate irrespective of the type of advertiser due to the

\footnotetext{
${ }^{5}$ The decision to use past seven days of data as a measure of past performance was based on a bidding strategy described to us by the advertiser that provided us data and by the search engine marketing firm that bids on behalf of this and other advertisers

${ }^{6}$ We use a log normal representation as the bids are non negative

${ }^{7}$ Search engines also use other factors such as design of the advertisement, landing page, etc. However, CTR is the primary input for the quality score
} 
sequential search by the consumers. In order to account for this, the performance is normalized for the position. As a result, an ad appearing in a lower position can be considered a better performer than a higher position ad even if its absolute CTR is lower as long as its position adjusted CTR is higher than that of the higher ranked ad. Past position also serves as a proxy for the intensity of the competition. Search engines use the most recent CTR information to determine the performance. Accordingly, we use the average CTR for the past week. We use the following reduced form equation to represent the ad position determined by the search engine for a keyword in the current period: ${ }^{8}$

$\ln \left(\operatorname{pos}_{\mathrm{kt}}\right)=\alpha^{\mathrm{k}} \mathrm{X}_{\mathrm{kt}}^{\alpha}+\varepsilon_{\mathrm{kt}}^{\alpha}$

$\alpha^{\mathrm{k}}=\Delta^{\alpha} \mathrm{z}_{\mathrm{k}}+\mathrm{u}_{\mathrm{k}}^{\alpha} \quad \mathrm{u}_{\mathrm{k}}^{\alpha} \sim \mathrm{N}\left(0, \mathrm{~V}^{\alpha}\right)$

where the covariates are as follows:

bid $_{\mathrm{kt}}$ is the bid in the current period

$\operatorname{pos}_{\mathrm{k}, \text { week-1 }}$ is the average position for keyword $\mathrm{k}$ for the past seven days,

$\mathrm{CTR}_{\mathrm{k}, \text { week-1 }}$ is the average CTR for keyword $\mathrm{k}$ for the past seven days,

$\delta_{\mathrm{kt}}^{\mathrm{m}}$ is an indicator function to control for the month,

\& a constant term.

In order to account for the correlation between the error terms for click-through rate, conversion rate, bid and position we use the following distribution

$\left[\begin{array}{c}\varepsilon_{\mathrm{kt}}^{\beta} \\ \varepsilon_{\mathrm{kt}}^{\theta} \\ \varepsilon_{\mathrm{kt}}^{\gamma} \\ \varepsilon_{\mathrm{kt}}^{\alpha}\end{array}\right] \sim \mathrm{N}(0, \Omega)$ where $\Omega=\left[\begin{array}{llll}\Omega_{11} & \Omega_{12} & \Omega_{13} & \Omega_{14} \\ \Omega_{21} & \Omega_{22} & \Omega_{23} & \Omega_{24} \\ \Omega_{31} & \Omega_{32} & \Omega_{33} & \Omega_{34} \\ \Omega_{41} & \Omega_{42} & \Omega_{43} & \Omega_{44}\end{array}\right]$

\footnotetext{
${ }^{8} \mathrm{We}$ use a log normal representation as the ad position is always positive
} 
We draw from the approach for linear hierarchical models (see Greg \& Allenby, 2005, p 71) to estimate the above equations as a recursive system. A similar approach has been adopted by Ghose \& Yang (2007).

\section{DATA \& ESTIMATION}

Our dataset is provided by a Search Engine Marketing firm that manages the sponsored search campaign on Google for a women's clothing brand. The data set consists of daily impressions, clicks, and conversions for 2,162 keywords over a 90-day period from April 2007 to June 2007.

Both prior industry studies and the extant academic literature (e.g., Johnson et al. 2004; Brynjolfsson, Dick, and Smith 2006) have shown that consumer search depth is very limited. For example, a survey conducted by iProspect (2006) showed that $81 \%$ of consumers do not search beyond the first two pages of search results. Because of this, we limit our analysis to those observations with an average position less than or equal to 10. This corresponds to the listings on the first page, and allows us to remove outliers from our dataset.

Further, since we wish to focus on the profitability of ads as a function of position, we are interested in only those advertisements that have to compete for these positions. These are associated with keywords that are popular among multiple advertisers. To focus our attention on "competitive" keywords we eliminate any keywords that are store-brand specific, keywords that have fewer than 10 clicks over the entire study period, and any keywords with an average CPC of less than $\$ 0.30$. We eliminate store brand specific keywords because, as an example, the keyword 'lane bryant dresses' is less likely to result in competitive bids than the keyword 'dresses.' The CPC restriction of $\$ 0.30$ was chosen based on Ganchev et al. (2007) who show that cost per click decays exponentially with the number of bidders. Using their decay factor we determine that the average CPC for the $10^{\text {th }}$ position for our dataset should be 32 cents. 
Table 1: Keyword Performance Summary Statistics

\begin{tabular}{|l|l|l|l|l|l|}
\hline \multicolumn{1}{|c|}{ Variable } & \multicolumn{1}{c|}{ Total } & \multicolumn{1}{c|}{ Mean } & \multicolumn{1}{c|}{ St. Dev. } & \multicolumn{1}{c|}{ Min } & \multicolumn{1}{c|}{ Max } \\
\hline Impressions & 882,4082 & 218.6 & 1027.15 & 1 & 47,070 \\
\hline Clicks & 264,375 & 6.55 & 34.58 & 0 & 989 \\
\hline Conversions & 2,537 & 0.063 & 0.41 & 0 & 14 \\
\hline Average Position & - & 5.24 & 2.15 & 1 & 10 \\
\hline Average CPC & - & 0.26 & 0.18 & 0 & 1 \\
\hline Length & - & 2.84 & 0.83 & 1 & 6 \\
\hline $\begin{array}{l}\text { Average } \\
\text { Position }\end{array}$ week-1 & & & & & \\
\hline CTR $_{\text {week-1 }}$ & - & 5.24 & 1.79 & 0 & 9.5 \\
\hline CV $_{\text {week-1 }}$ & - & 0.036 & 0.029 & 0 & 0.25 \\
\hline Profit $_{\text {week-1 }}$ & - & $4.9 \mathrm{e}-04$ & 0.0016 & 0 & 0.029 \\
\hline & & 2.61 & 13.68 & -18.6 & 198.7 \\
\hline
\end{tabular}

These sequential restrictions result in the removal of 57, 711 observations, and 591 keywords. Our final sample contains 40,358 observations for 803 keywords ${ }^{9}$. We note that our qualitative results are not sensitive to relaxing the criteria for clicks and CPC mentioned above. However, the magnitude of the coefficients are smaller due to the fact that the extended dataset includes keywords for which only a few competing advertisements appear, as compared to the larger number of competitors for competitive keywords. Summary statistics for our final sample are given in Table 1.

The position reported for any keyword is the average position on a given day. The position usually varies within a day because advertisers may choose a "broad match" for their keywords meaning that the ad is shown if a consumer enters a broadly related keyword. For example, the ad for keyword "dress" maybe shown if the consumer types "red dress" or "green dress". The competitors and their bids may be different for these two queries causing the position to vary. On the other hand, an "exact match" (ad is shown only if the query is exactly the same) fixes the competitors rather than letting it vary based on consumer query. Our result holds for the subset

\footnotetext{
${ }^{9}$ We have separately verified that our final set of keywords do generate sufficient number of advertisements.
} 
of keywords with "exact match" option selected as well. Another reason for the position to vary is that competitors may change their bids multiple times within a day. While firms change bid periodically, typically weekly and sometimes even daily, we do not find significant intra-day variation in ad position for keywords with exact match. ${ }^{10}$

\section{Estimation Method}

We estimate the model using a Bayesian approach, applying Markov chain Monte Carlo sampling due to the non-linear characteristics of our model (Rossi \& Allenby, 2005). For our dataset, Bayesian analysis offers several advantages over standard econometric analysis. First, several keywords in our dataset have sparse observations. For example, about $60 \%$ of our keywords have no conversion data in the time period under consideration or are missing data for several positions. Classical techniques, which rely on the asymptotic properties of large samples, may give biased estimates in this context. As a result of these and related factors, Bayesian analysis is preferable to classical asymptotic techniques to model sponsored search data (see Rutz and Bucklin, 2006 for additional discussion). For a discussion of the priors and conditional posteriors of this model, please refer to the Technical Appendix A1. For the HB Models, we run the MCMC simulation for 20,000 draws and discard the first 5,000 as burn-in.

In order to ensure that our parameter estimates are accurate we have simulated the clicks, conversions, bids and positions using our estimates. By repeating the estimation with this simulated dataset we were able to recover our parameter estimates. This indicates our parameters are fully identified.

\footnotetext{
${ }^{10}$ We have separately verified this for a select set of keywords with exact match by monitoring the relative ad positions across multiple queries in a day.
} 


\section{RESULTS}

\section{$C T R \& C V I$}

Table 2 provides the mean values for the posterior distribution of the $\Delta^{\beta} \& \Delta^{\theta}$ matrix from equations 2 and 5. Both pos and $\operatorname{pos}^{2}$ terms are significant for CVI as well as CTR. For the average keyphrase length in our data of 2.84 , position has a negative effect on CTR i.e. CTR decreases as we move down the ranked list of ads. For CVI, we find that the coefficient for pos is positive and dominates the $\operatorname{pos}^{2}$ term (which has a negative coefficient) for the first few positions for longer keyphrases. Thus, the conversion rate per impression initially increases and then decreases as we move down the ranked list of ads. Table 2 also reports the unobservable heterogeneity in terms of the square root of the diagonal terms in the covariance matrix in equations 2 and 5. The large values indicate that there is significant heterogeneity across the keywords, underscoring the importance of accounting for heterogeneity in this environment.

Table 2: Estimates for the CVI and CTR

\begin{tabular}{|c|c|c|c|c|c|c|}
\hline \multirow{5}{*}{$\begin{array}{l}\text { Const } \\
\text { pos } \\
(\text { pos)^2 }\end{array}$} & \multicolumn{3}{|c|}{ CVI } & \multicolumn{3}{|c|}{ CTR } \\
\hline & Intercept & Length & $\begin{array}{c}\text { unobs } \\
\text { het }\end{array}$ & Intercept & Length & $\begin{array}{c}\text { unobs } \\
\text { het }\end{array}$ \\
\hline & $-8.53(0.46)^{\star * *}$ & $-0.38(0.14)^{\star \star *}$ & 0.11 & $-3.35(0.25)^{* * *}$ & $0.03(0.11)$ & 0.97 \\
\hline & $-0.47(0.20)^{\star *}$ & $0.27(0.05)^{\star \star \star}$ & 0.11 & $0.95(0.35)^{\star \star \star}$ & $-0.26(0.12)^{\star *}$ & 4.03 \\
\hline & $-0.03(0.01)^{* * *}$ & $-0.01(0.00)^{\star * *}$ & 0.16 & $-0.27(0.05)^{* * *}$ & $0.07(0.02)^{* * *}$ & 0.94 \\
\hline
\end{tabular}

We also observe that the keyphrase length has a significant impact on CTR and CVI. Figure 2 shows the expected CTR \& CVI as a function of position for different keyphrase lengths. CTR is higher for the short keyphrases at top positions. Shorter keyphrases are generic with very high search volume. If the keyphrases are related to commercial products, one can expect consumers to at least evaluate the top ads. This can translate to higher click through rate for the top ads for shorter keyphrases as compared to the longer keyphrases. However, shorter keyphrases may 
result in greater mismatch between the user intent and the displayed ad. As a consequence, users may search fewer ads for shorter keyphrases leading to a low CTR for shorter keyphrases at lower positions. Longer key phrases allow search engines to better infer the consumer's search intention resulting in a better match between the user query and displayed results. Additionally, longer keyphrases can also reflect a higher purchase intent in which case the consumer may evaluate more ads. This results in longer keyphrases having a higher CTR at lower position as compared to shorter keyphrases and a higher overall CVI as compared to shorter keyphrases (Figure 2).
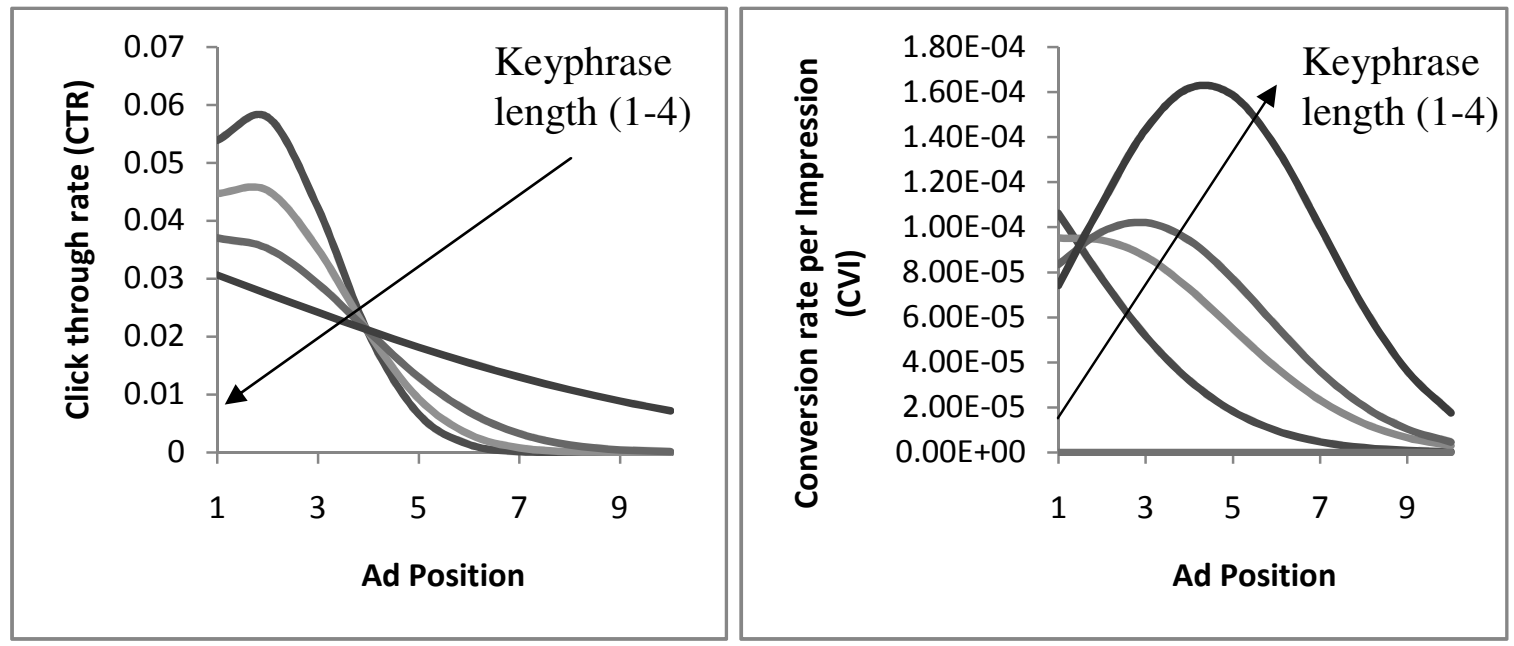

Figure 2: Expected CTR \& CVI as a function of position

We have also considered placement effects (i.e. separately examining keywords which regularly have ads appearing above the organic results versus those which always have ads on the right side). We find that keywords with placement above the organic results have better overall performance. However, our results hold for both types of keywords i.e. revenue is higher at lower positions for both types of keywords. 


\section{Advertiser's Bid and Ad Position}

Advertiser's Bid: Table 3 provides the mean values for the posterior distribution of the $\Delta^{\gamma}$ matrix from equation 8. We can see that all the coefficients are significant. However, the magnitude of coefficient for CTR is highest, indicating that the advertiser places emphasis on the past click through rate in deciding the current bids. This is reasonable as it is very difficult to measure the conversion performance due to the sparse nature of the observed data.

Table 3: Estimates for the Advertiser's Bid

\begin{tabular}{|l|l|l|r|}
\hline Variables & \multicolumn{1}{|c|}{ Intercept } & \multicolumn{1}{c|}{ Length } & unobs het \\
\hline Const & $-1.09(1.4 \mathrm{E}-03)^{* * *}$ & $0.05(6.2 \mathrm{E}-04)^{\star * *}$ & 0.113 \\
PoS $_{\text {week-1 }}$ & $0.001(2.4 \mathrm{E}-04)^{\star * *}$ & $0.01(8.7 \mathrm{E}-05)^{\star * *}$ & 0.122 \\
CTR $_{\text {week-1 }}$ & $0.17(1.5 \mathrm{E}-02)^{\star * *}$ & $-0.10(1.0 \mathrm{E}-02)^{\star * *}$ & 0.112 \\
CV $_{\text {week-1 }}$ & $0.02(1.2 \mathrm{E}-03)^{\star * *}$ & $-0.01(1.2 \mathrm{E}-03)^{\star * *}$ & 0.112 \\
Profit & & $0.01(1.9 \mathrm{E}-04)^{* * *}$ & 0.113 \\
\hline
\end{tabular}

Ad position: Table 4 provides the mean values for the posterior distribution of the $\Delta^{\alpha}$ matrix from equation 9.

Table 4: Estimates for the Ad Position

\begin{tabular}{|l|l|l|r|}
\hline Variables & \multicolumn{1}{|c|}{ Intercept } & \multicolumn{1}{c|}{ Length } & unobs het \\
\hline Const & $1.37(4.0 \mathrm{E}-03)^{\star * *}$ & $-0.29(7.6 \mathrm{E}-04)^{\star * *}$ & 0.120 \\
Bid & $-0.51(6.1 \mathrm{E}-03)^{\star * *}$ & $0.12(1.1 \mathrm{E}-03)^{\star \star *}$ & 0.120 \\
Pos & $0.08(5.7 \mathrm{E}-04)^{\star * *}$ & $0.04(1.4 \mathrm{E}-04)^{\star * *}$ & 0.120 \\
CTR $_{\text {week }-1}$ & $-1.03(9.3 \mathrm{E}-02)^{\star * *}$ & $0.22(1.1 \mathrm{E}-02)^{\star \star *}$ & 0.110 \\
\hline
\end{tabular}

Higher bids lead to higher current position. Similarly higher CTR leads to higher current position. This is reasonable as both bids and CTR are the primary inputs used to compute the ad rank. The past position should moderate the effect of CTR i.e. same CTR at lower past position should lead to higher current position. However, we observe that the current position is positively correlated with the past position. This is because the past position also serves as a 
proxy for advertiser's performance relative to other advertisers. For a given bid, a lower past position indicates that there are more competitors with a higher performance and as a result, the ad for the keyword is more likely to get a lower current position.

Table 5: Estimates for the Covariance Matrix $\Omega$

\begin{tabular}{l|l|l|l|l|}
\multicolumn{1}{l}{ CVI } & \multicolumn{1}{l}{ CTR } & \multicolumn{1}{l}{ Bid } & \multicolumn{1}{l}{ Ad Position } \\
\cline { 2 - 5 } CVI & $18.2(2.56)^{* * *}$ & $0.96(0.24)^{* * *}$ & $2.04 \mathrm{E}-3(1.94 \mathrm{E}-3)$ & $1.69 \mathrm{E}-2(9.18 \mathrm{E}-3)^{*}$ \\
CTR & & $1.20(4.55)^{* * *}$ & $-1.19 \mathrm{E}-2(1.92 \mathrm{E}-3)^{* * *}$ & $3.71 \mathrm{E}-3(5.41 \mathrm{E}-3)^{*}$ \\
Bid & & $5.14 \mathrm{E}-3(3.55 \mathrm{E}-5)^{* * *}$ & $5.43 \mathrm{E}-4(7.80 \mathrm{E}-5)^{* * *}$ \\
Ad Position & & & & $4.47 \mathrm{E}-2(3.11 \mathrm{E}-4)^{* * *}$ \\
\cline { 3 - 5 } & & &
\end{tabular}

Finally, Table 5 shows unobserved covariance between the conversions, clicks, bids and ad positions. Covariance between the unobservables for CVI and CTR is significant. We can also see that the covariance between the unobservables for CVI and other measures is not statistically significant. However, covariance between the unobservables for CTR and bids is statistically significant. This again establishes that the advertiser is not placing much value on the conversions in deciding its bids. One can expect a similar behavior from other advertisers. Similarly, the ranking by search engine is related to the unobservables for bids only. As a consequence, the position determined by the search engine based on the bids of the advertisers has little influence on the actual conversion rate per impression.

\section{Performance as a function of position}

Cost: In order to assess the impact of ad position on profitability, we need to know the impact of position on the cost of the keywords. We know that CPC should decrease with position due the generalized second price auction mechanism. Therefore it follows immediately from Figure 2 that the top position is not the most profitable for longer keyphrases. 
The practitioner and academic literature have established that CPC decays exponentially with position. In order to determine the true cost behavior in these auctions we need information about the competing bids for each keyword $k$ at time $t$. However, as mentioned above, this information is not available to the advertisers. Additionally, for a given keyword advertisers change their bids over time. As a consequence, the cost for a keyword can change with time for the same position. We use the relationship between the search engine rank and the advertiser's bid (equation 9) to determine the cost. As mentioned earlier, the past performance (position adjusted CTR) serves as a proxy for the competition and we can expect different cost curves depending on the past position of the advertiser. For example, a past position of one would indicate that the advertiser is not facing too much competition, can bid a low amount and still retain its position. For a given bid and position $j$, we assume that the actual cost per click (CPC) is the bid for the position $j+1$. $^{11}$
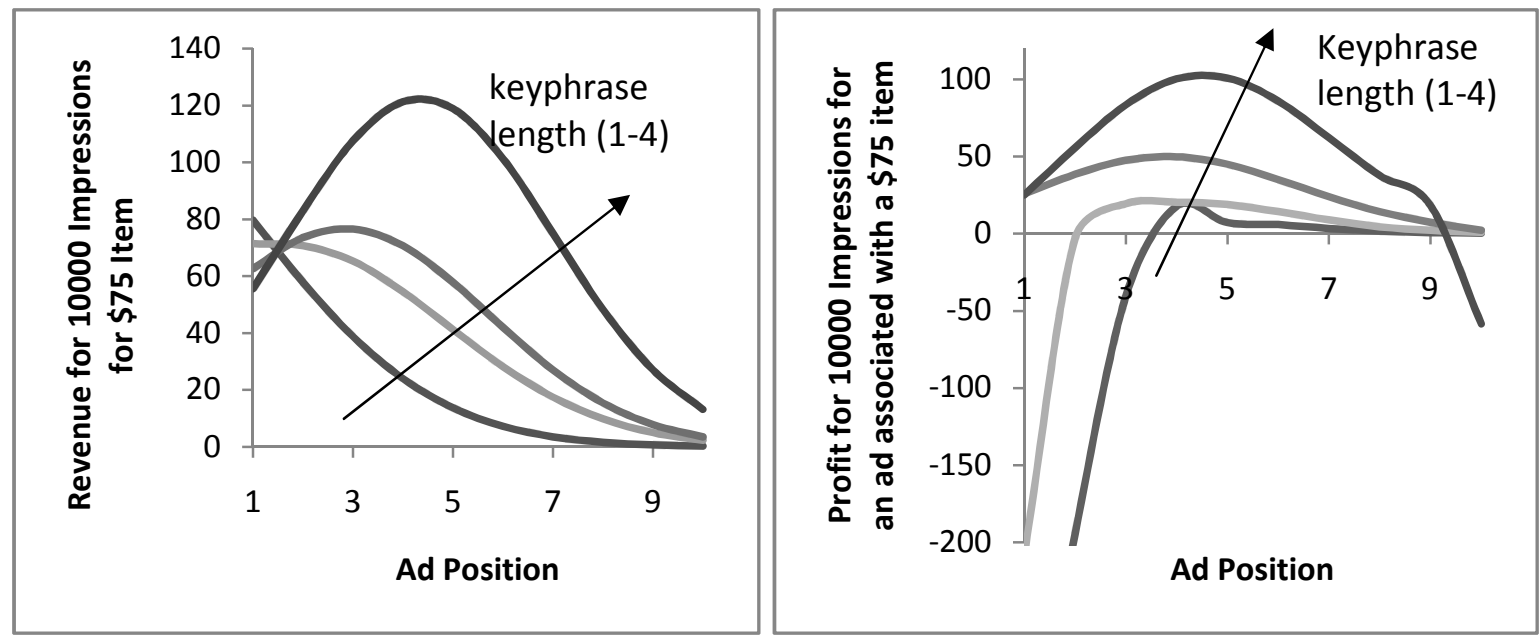

Figure 3: Revenue \& Profit as a function of Position

\footnotetext{
${ }^{11}$ The actual cost for position $\mathrm{j}$ is determined using a generalized second prize auction and depends on the bid and the position adjusted CTR of the advertiser in position $\mathrm{j}+1$. Our calculation assumes that the position adjusted CTRof the advertiser in position $\mathrm{j}+1$ is same as that for our advertiser. This should only affect the scale and not the decay for the cost function
} 
Revenue \& Profit: Revenue can be calculated using the CVI as a function of position for different keyphrase lengths (table 2). Using the above method to determine the cost, we can calculate the expected profit using equation 1 and the mean $\Delta$ values from tables $2 \& 4$. We assume that cost is the amount required to maintain the current position. Our results are graphed in Figure 3 using a value of $\$ 75$ (average value of goods sold in our sample) for each unit of the advertised good sold, and with 10,000 impressions. We find that for our advertiser, lower ad positions generate higher revenue for longer keyphrases due to non-monotonic conversion rate. Thus, our results show that while click through rate for ads decreases with position, conversion rate as well as revenue demonstrate non-monotonic behavior (i.e. both the conversion rate and the revenue increase for the first few positions and then decrease) for longer keyphrases. Conventional wisdom suggests that with sequential evaluation, items shown earlier on would be considered by a larger proportion of consumers and would have a higher demand. Although the decrease in CTR with position is consistent with that view, the non-monotonicity in revenue is counter to this view.

As a consequence, lower ad positions are often more profitable than the top positions for longer keyphrases. This is true even for shorter length keyphrases which always have a decreasing conversion rate with position. This is because the cost is decaying at a faster rate as compared to the conversion rate. There has been some evidence (Kitts \& Leblanc, 2004) that the bid efficiency is not the highest for the top position. However, to the best of our knowledge, our study is the first one to rigorously prove that this indeed is the case. For longer keyphrases with non-monotonic conversion rates, the profit curves are shifted further to the right due to the declining costs with position. 


\section{DISCUSSION \& CONCLUSION}

In this paper, we analyze the impact of position on the revenues and profitability of sponsored search advertisements that appear alongside regular algorithmic search results in search engines. A widely held belief in the industry is that the higher the ad placement the better the performance. Most of these observations are based primarily on an observed exponential decay in the click through rate (CTR) of the advertisements as a function of their position as opposed to a more careful analysis of conversions and revenues.

We analyze the impact of position on ad profitability using a unique dataset obtained from a Search Engine Marketing (SEM) firm. This dataset documents the daily clicks, conversions, and costs for a campaign sponsored by one of the firm's clients. Consistent with the prior literature, our study confirms that CTR decreases rapidly with the rank of the ad. However, for advertisers interested in maximizing revenues or profit (as opposed to exposure benefit), this only tells part of the story. Our results also show that an advertisement's revenue initially increases with position for longer keyphrases. As the ranking mechanism used by the search engines does not account for conversion rate, ads placed in the top position do not always maximize revenues. We also show that even for keyphrases where the conversion rate decreases with the ad position, the top position may not be profit maximizing due to a higher rate of decrease in cost with position.

These findings are important to the industry as advertisers are currently engaged in intense bidding wars to secure the top positions in sponsored search results. Our results suggest that these bidding strategies may be based on faulty assumptions about the relationship between click-through rate, cost per click, and conversion probability as a function of position. Our results suggest that, at present, advertisers seeking to maximize transactional benefits are often better off in the short term by placing less weight on obtaining top positions. It is important to 
note that this is not an equilibrium argument and the strategy will not work if all advertisers follow the same approach in the long run. However, it does emphasize the importance of tracking conversions.

Our study also sheds light on consumer behavior in sponsored search environments. It is clear that click through rates at top positions are considerably higher than those at lower positions. This suggests that most consumers conduct limited search and have small consideration sets. We also find that both conversion rate and revenues first increase and then decrease with ad position for longer keyphrases. The initial increase with position suggests that consumers with higher purchase intent may be evaluating at least a few positions before making their purchase decisions. A subsequent decrease in the conversation rate and revenues at lower positions similarly suggests that consumers with high purchase intent stop their product search after the first few slots and only consumers seeking information may be clicking the advertisements at these lower positions. If this is the case, then placing ads at intermediate positions may be an effective way to reach buying consumers without paying more for the top positions.

Finally, our study points to potential inefficiencies in the auction mechanisms used by popular search engines. If advertisers with the best combination of bid and CTR are assigned the top position and lower positions generate higher revenues then this may well be doing them a disservice. One alternative approach available to search engines is to invest in technologies to track consumer action post-click and to charge advertisers per conversion (also known as Pay Per Action or PPA auctions). PPA auctions are currently being tested by several search engines. ${ }^{12}$

\footnotetext{
${ }^{12}$ See "Google launches test of pay-per-action ads", Information Week, March 2007. Retrieved from http://www.informationweek.com/news/internet/search/showArticle.jhtml?articleID=198500474
} 
As with any empirical analysis there are several limitations of our study. Our analysis is based on data from one industry. It would be useful for future studies to examine other industry verticals. A further limitation is that, lacking appropriate data, we were forced to use only the advertiser specific information to determine the cost decay. This is because sponsored search auctions are now implemented as closed auctions and the true cost of securing other positions is not known. Access to bid data from other advertisers can help increase the accuracy of our findings. However, we do not expect the direction of findings to reverse with such analysis. This is because we observed an inverse U-shaped relationship between revenues and ad position for longer keyphrases. Given that average CPC associated with top positions is greater than that with lower positions, by definition, the inverse-U relationship will only be strengthened when analyzing profits. Similarly, while our results explain the impact of position on click-through rate, conversion rate, and revenue for the buying consumers, the aggregate nature of our data limits our ability to account for the actions of individual consumers. This calls for future research using click stream data to empirically evaluate the behavior of different types of consumers in sponsored search.

An additional limitation is that our analysis of conversions is based on measurements conducted by the SEM firm wherein consumer action is tracked during the entire search session. This is potentially problematic because, consumers may click on an ad, visit the advertiser's landing page without converting but return on a later day (even using a different search engine query) to then buy the product. In these instances, the future purchases are not properly attributed to the original keyword. There can also be a spillover effect of competitive keywords on the branded keywords i.e. the consumers may engage in the search using more popular keywords and then buy the product at a later point in time using a branded keyword that will show them the 
relevant brand site (Rutz and Bucklin, 2007). Future research should measure these effects as a function of position. Finally, our analysis has only focused on transactional benefits from advertising. We believe this is a reasonable approach in our data setting. However in other settings, non-transactional benefits such as branding and awareness may be more important to advertisers. Analysis of sponsored search strategies in such settings would be a fruitful area for future research.

\section{References}

Animesh, A., V. Ramachandran, and S. Viswanathan. (2006). Quality Uncertainty and Adverse Selection in Online Sponsored Search Markets. Proc. 27th International Conference on Information Systems (ICIS), Milwaukee, Wisconsin.

Arbatskaya, M. (2007). Ordered Search. Rand Journal of Economics, 38(1), 119-126.

Bearden, J. N., Rapoport, A., Murphy, R. O. (2006). Sequential Observation and Selection with Rank-Dependent payoffs: An Experimental Study. Management Science, 52(9), 1437-1449.

Borgs, C., Chayes, J., Etesami, O., Immorlica, N., Jain, K. and Mahadian, M. (2007). Dynamics of Bid Optimization in Online Advertisement Auctions

Brunel, F. F., Nelson, M. R. (2003). Message Order Effects and Gender Differences in Advertising Persuasion. Journal of Advertising Research, 43(3), 330-341.

Brynjolfsson, E., A.A. Dick, M.D. Smith. (2006). A Nearly Perfect Market? Differentiation vs. Price in Consumer Choice. Working Paper, Carnegie Mellon University, Pittsburgh, PA. 
Cary, M., Das, A., Edelman, B., Giotis, I., Heimerl, K., Karlin, A., Mathieu, C., and Schwarz, M. (2007). Greedy Bidding Strategies for Keyword Auctions. ACM Conference on Electronic Commerce Series, 2007.

Chakravarti, A., Janiszewski, C., and Ulkumen, G. (2006). The Neglect of Prescreening Information. Journal of Marketing Research, 63(11), 642-653.

Chen, Y. \& He, C. (2006). Paid Placement: Advertising and Search on the Internet, Working Paper.

Dellarocas, C. and Viswanathan, S. (2007). The Holy Grail of Advertising? Quality Signaling and Revenue Implications of Pay-per-Performance Advertising. Workshop on Information Systems and Economics (WISE), Montreal, Quebec, Canada, December 2007.

Diehl, K., Kornish, L. J., and Lynch J, G. (2003). Smart Agents: When Lower Search Cost for Quality Information Increases Price Sensitivity. Journal of Consumer Research, 30 (6), 56-71.

Edelman, B., Ostrovsky, M. (2007). Strategic Bidder Behavior in Sponsored Search Auctions. Decision Support Systems, 43, 192-198.

Edelman, B., Ostrovsky, M. \& Schwarz, M. (2007). Internet Advertising and the Generalized Second Price Auction: Selling Billions of Dollars Worth of Keywords, American Economic Review, forthcoming March 2007.

Esteban, L., Hernández, J.M., and Moraga-Gonzalez, J.L. (2006). Customer Directed Advertising and Product Quality. Journal of Economics and Management Strategy, 15(4), 943968. 
Feldman, J., Muthukrishnan, S., Pal, M., and Stein, C. Budget optimization in search-based advertising auctions. Proceedings of the 8th ACM Conference on Electronic Commerce, 2007.

Feng, F., Bhargava, H., and Pennock, D. 2007. Implementing Sponsored Search in Web Search Engines: Computational Evaluation of Alternative Mechanisms. Informs Journal on Computing, 19(1),137-148.

Ganchev, K., Kulesza, A., Tan, J., Gabbard, R., Liu, Q., Kearns, M. (2007). Empirical Price Modeling for Sponsored Search. Working Paper.

Ghose, A. and Yang, S. (2007). Towards Empirically Modeling Consumer and Firm Behavior in Search Engine Advertising. Workshop on Information Systems and Economics (WISE), 2007.

Goldfarb, A and Tucker, C. (2007). Search Engine Advertising: Pricing Ads to Context. NET Institute Working Paper \#07-23.

Häubl, G. and Valerie, T. (2000). Consumer Decision Making in Online Shopping Environments: The Effects of Interactive Decision Aids. Marketing Science, 19 (Winter), 4-21

Hauser, J. R. and Wernerfelt, B (1990). An Evaluation Cost Model of Evoked Sets. Journal of Consumer Research, 16(3), 393-408.

Hoque, A.Y., and Lohse, G.L (1999) An Information Search Cost Perspective for Designing Interfaces for Electronic Commerce. Journal of Marketing Research, 36(3), 387-394.

Hosanagar, K., and Cherapanov, V. 2008. Optimal Bidding in Stochastic Budget Constrained Slot Auctions. Proc. Of ACM Conference on Electronic Commerce, Chicago, July 2008. 
iProspect survey 2006. iProspect Search Engine User Behavior Study.

http://www.iprospect.com/premiumPDFs/WhitePaper_2006_SearchEngineUserBehavior.pdf

Janiszewski, C. (1998). The influence of display characteristics on visual exploratory search behavior. Journal of Consumer Research, 25, 290-301.

Johnson, E. J., Moe, W. W., Fader, P. S., Bellman, S., Lohse, G.L. 2004. On the depth and dynamics of online search behavior. Management Science, 50(3), 299-308.

Kitts, B., and LeBlanc, B. 2004. Optimal Bidding on Keyword Auctions, Electronic Markets,

Kitts, B., Laxminarayan, P., LeBlanc, B., and Meech, R. (2005).A formal analysis of search auctions including predictions on click fraud and bidding tactics. Workshop on Sponsored Search Auctions, June 2005.

Lahaie, S.; Pennock, D. M. (2007). Revenue Analysis of a Family of Ranking Rules for Keyword Auctions. ACM Conference on Electronic Commerce (EC).

Lohse, G. L. (1997). Consumer Eye Movement Patterns on Yellow Pages Advertising. Journal of Advertising, 26(1), 61-73.

Misra, P., Pinker, E., Kauffman A. R. (2006). An Empirical Study of Search Engine Advertising Effectiveness. Workshop on Information Systems and Economics (WISE), 2006.

Moe, W. W. 2003. Buying, searching, or browsing: Differentiating between online shoppers using in-store navigational clickstream. J. Consumer Psych. 13(1, 2) 29-40.

Moe, W. W. and Fader, P. S. (2004). Dynamic Conversion Behavior at e-Commerce Sites. Management Science, 50 (3), 326-335. 
Montgomery, A. L., Hosanagar,K., Krishnan, R. and Clay. K. B. (2004). Designing a Better Shopbot. Management Science, 50(2), 189-206.

Montgomery, A. L., Li, S., Srinivasan, K., and Liechty, J. C. (2004), "Modeling Online Browsing and Path Analysis Using Clickstream Data", Marketing Science, 23(4), 579-595.

Muthukrishnan, S., Pal, M., and Svitkina, Z. Stochastic models for budget optimization in search-based. Proceedings of the World Wide Web conference, 2007.

Rhodes, E.W., Teferman, N. B., Cook, E. and Schwartz, D. (1979). T-Scope tests of yellow pages advertising. Journal of Advertising Research, 19, 49-52.

Rossi, P. E. \& Allenby, G. M.(2003). Bayesian Statistics and Marketing. Marketing Science, 22(3), 304-328.

Rossi, P. E. \& Allenby, G. M. (2005). Bayesian Statistics and Marketing. John Wiley and Sons.

Rutz, O. \& Bucklin, R (2006). A Model of individual Keyword Performance in Paid Search Advertising. Working Paper.

Rutz, O. \& Bucklin, R (2007). From Generic to Branded: A Model of Spillover Dynamics in Paid Search Advertising. Working Paper.

Target Marketing (2006). Bid Fight. February, 29(2), 39-42.

Varian, H.R (2006). Position Auctions. International Journal of Industrial Organization, 2006.

Villas-Boas, J.M. and R.S. Winer (1999). Endogeneity in Brand Choice Models. Management Science, 45, 1324-1338. 
Wall Street Journal (2007). Keywords: a Growing Cost for News Sites; Media Firms Place Bids To Secure Top Positions With Search Engines. April 30, pg B6.

Weber, T.A. and Z. Zheng (2007). A Model of Search Intermediaries and Paid Referrals. Information Systems Research, 18 (4), 414-436.

Zhang, J., Fang, X. and Sheng, ORL. (2007). Online consumer search depth: Theories and new findings. Journal of Management Information Systems, 23(3), 71-75. 


\section{Technical Appendix A1}

The MCMC algorithm is describe below:

Step I: DrawU $\mathrm{ut}_{\mathrm{kt}}^{\mathrm{CVI}} \& \mathrm{U}_{\mathrm{kt}}^{\mathrm{CTR}}$

We use random walk Metropolis-Hastings algorithm for sampling $\mathrm{U}_{\mathrm{kt}}=\left(\mathrm{U}_{\mathrm{kt}}^{\mathrm{CVI}}, \mathrm{U}_{\mathrm{kt}}^{\mathrm{CTR}}\right)($ Rossi \& Allenby, 2005)

$\mathrm{U}_{\mathrm{kt}}^{\text {new }}=\mathrm{U}_{\mathrm{kt}}^{\text {old }}+\delta$ where $\delta \sim \mathrm{N}(0,0.01 \mathrm{I})$

The draws are accepted with a probability $\alpha$ where

$$
\begin{gathered}
\alpha=\min \left[\frac{\exp \left[-\frac{1}{2}\left(\mathrm{U}_{\mathrm{kt}}^{\mathrm{new}}-\mathrm{Y}_{\mathrm{kt}}-\mathrm{e}\right)^{\prime} \mathrm{A}\left(\mathrm{U}_{\mathrm{kt}}^{\mathrm{new}}-\mathrm{Y}_{\mathrm{kt}}-\mathrm{e}\right)\right] \mathrm{l}\left(\mathrm{U}_{\mathrm{kt}}^{\mathrm{new}}\right)}{\exp \left[-\frac{1}{2}\left(\mathrm{U}_{\mathrm{kt}}^{\mathrm{old}}-\mathrm{Y}_{\mathrm{kt}}-\mathrm{e}\right)^{\prime} \mathrm{A}\left(\mathrm{U}_{\mathrm{kt}}^{\mathrm{old}}-\mathrm{Y}_{\mathrm{kt}}-\mathrm{e}\right)\right] \mathrm{l}\left(\mathrm{U}_{\mathrm{kt}}^{\mathrm{old}}\right)}, 1\right] \\
\mathrm{l}\left(\mathrm{U}_{\mathrm{kt}}\right)=\prod_{\mathrm{k}=1}^{\mathrm{K}} \prod_{\mathrm{t}=1}^{\mathrm{T}}\left(\Lambda_{\mathrm{kt}}^{\mathrm{CVI}} \cdot \Lambda_{\mathrm{kt}}^{\mathrm{CTR}}\right)^{\operatorname{Orders}_{\mathrm{kt}}\left(\left(1-\Lambda_{\mathrm{kt}}^{\mathrm{CVI}}\right) \cdot \Lambda_{\mathrm{kt}}^{\mathrm{CTR}}\right)^{\mathrm{Clicks}_{\mathrm{kt}}-\text { Orders }_{\mathrm{kt}}}((1} \\
\left.\left.-\Lambda_{\mathrm{kt}}^{\mathrm{CVI}}\right)\left(1-\Lambda_{\mathrm{kt}}^{\mathrm{CTR}}\right)\right)^{\text {Impressions }_{\mathrm{kt}}-\mathrm{Clicks}_{\mathrm{kt}}}
\end{gathered}
$$

$\mathrm{e}=\left[\begin{array}{c}\mathrm{e}_{\mathrm{kt}}^{1} \\ \mathrm{e}_{\mathrm{kt}}^{2}\end{array}\right]$ where $\mathrm{e}_{\mathrm{kt}}^{1}=\ln \left(\mathrm{bid}_{\mathrm{kt}}\right)-\gamma^{\mathrm{k}} \mathrm{X}_{\mathrm{kt}}^{\gamma} \& \mathrm{e}_{\mathrm{kt}}^{2}=\ln \left(\operatorname{pos}_{\mathrm{kt}}\right)-\alpha^{\mathrm{k}} \mathrm{X}_{\mathrm{kt}}^{\alpha}$

$$
\mathrm{A}^{-1}=\mathrm{W}_{11}-\mathrm{W}_{12} \mathrm{~W}_{22}^{-1} \mathrm{~W}_{21}
$$

$\mathrm{W}_{11}=\left[\begin{array}{ll}\Omega_{11} & \Omega_{12} \\ \Omega_{21} & \Omega_{22}\end{array}\right], \mathrm{W}_{22}=\left[\begin{array}{ll}\Omega_{33} & \Omega_{34} \\ \Omega_{43} & \Omega_{44}\end{array}\right], \mathrm{W}_{12}=\mathrm{W}_{21}=\left[\begin{array}{ll}\Omega_{13} & \Omega_{14} \\ \Omega_{23} & \Omega_{24}\end{array}\right]$

Step II: Draw $b_{k}=\left[\beta^{\mathrm{k}} \theta^{\mathrm{k}} \gamma^{\mathrm{k}} \alpha^{\mathrm{k}}\right]$

We define

$$
\begin{aligned}
& \mathrm{X}_{\mathrm{k}}=\left[\begin{array}{ccrr}
\mathrm{X}_{\mathrm{k}}^{\beta^{\prime}} & 0 & 0 & 0 \\
0 & \mathrm{X}_{\mathrm{k}}^{\theta^{\prime}} & 0 & 0 \\
0 & 0 & \mathrm{X}_{\mathrm{k}}^{\gamma^{\prime}} & 0 \\
0 & 0 & 0 & \mathrm{X}_{\mathrm{k}}^{\alpha^{\prime}}
\end{array}\right] \quad \mathrm{y}_{\mathrm{k}}=\left[\begin{array}{c}
\mathrm{U}_{\mathrm{k}}^{\mathrm{CVI}} \\
\mathrm{U}_{\mathrm{k}}^{\mathrm{CTR}} \\
\ln \left(\mathrm{bid}_{\mathrm{k}}\right) \\
\ln \left(\mathrm{pos}_{\mathrm{k}}\right)
\end{array}\right], \mathrm{V}=\left[\begin{array}{cccc}
\mathrm{V}^{\beta} & 0 & 0 & 0 \\
0 & \mathrm{~V}^{\theta} & 0 & 0 \\
0 & 0 & \mathrm{~V}^{\gamma} & 0 \\
0 & 0 & 0 & \mathrm{~V}^{\alpha}
\end{array}\right], \overline{\mathrm{b}_{\mathrm{k}}}=\left[\begin{array}{c}
\Delta^{\beta} \mathrm{z}_{\mathrm{k}} \\
\Delta^{\theta} \mathrm{z}_{\mathrm{k}} \\
\Delta^{\gamma} \mathrm{z}_{\mathrm{k}} \\
\Delta^{\alpha} \mathrm{z}_{\mathrm{k}}
\end{array}\right] \\
& \mathrm{Q}_{\mathrm{k}}=\left[\left(\mathrm{x}_{\mathrm{k}}^{\prime} \Omega \mathrm{x}_{\mathrm{k}}\right)^{-1}+\mathrm{V}^{-1}\right]^{-1} \& \widetilde{\mathrm{b}_{\mathrm{k}}}=\mathrm{Q}_{\mathrm{k}}\left[\mathrm{x}_{\mathrm{k}}^{\prime} \Omega^{-1} \mathrm{y}_{\mathrm{k}}+\mathrm{V}^{-1} \overline{\mathrm{b}_{\mathrm{k}}}\right]
\end{aligned}
$$


Then $b_{k} \sim N\left(\widetilde{b_{k}}, Q_{k}\right)$

Step III: Draw $\Omega$

$\Omega \sim \mathrm{IW}\left(v+\mathrm{N}, \sum_{\mathrm{k}=1}^{\mathrm{K}} \sum_{\mathrm{t}=1}^{\mathrm{T}} \mathrm{Y}_{\mathrm{kt}}^{\prime} \mathrm{Y}_{\mathrm{kt}}+\mathrm{S}\right) \quad$ where $\mathrm{Y}_{\mathrm{kt}}=\left[\begin{array}{c}\mathrm{U}_{\mathrm{kt}}^{\mathrm{CVI}}-\beta^{\mathrm{k}} \mathrm{X}_{\mathrm{kt}}^{\beta} \\ \mathrm{U}_{\mathrm{kt}}^{\mathrm{CTR}}-\theta^{\mathrm{k}} \mathrm{X}_{\mathrm{kt}}^{\theta} \\ \ln \left(\mathrm{bid}_{\mathrm{kt}}\right)-\gamma^{\mathrm{k}} \mathrm{X}_{\mathrm{kt}}^{\gamma} \\ \ln \left(\operatorname{pos}_{\mathrm{kt}}\right)-\alpha^{\mathrm{k}} \mathrm{X}_{\mathrm{kt}}^{\alpha}\end{array}\right], \mathrm{N}=$ No of observations,

$v=10, \mathrm{~S}=10 \mathrm{I}$

Step IV: Draw $V^{\beta} V^{\theta} V^{\gamma} V^{\alpha}$

$\mathrm{V}^{\beta} \sim \mathrm{IW}\left(\nu+\mathrm{N}, \sum_{\mathrm{k}=1}^{\mathrm{K}}\left(\beta^{\mathrm{k}}-\Delta^{\beta} \mathrm{z}_{\mathrm{k}}\right)^{\prime}\left(\beta^{\mathrm{k}}-\Delta^{\beta} \mathrm{z}_{\mathrm{k}}\right)+\mathrm{S}\right)$

where $\mathrm{N}=$ No of keywords, $v=10, \mathrm{~S}=10 \mathrm{I}$

$\mathrm{V}^{\theta} \sim \mathrm{IW}\left(v+\mathrm{N}, \sum_{\mathrm{k}=1}^{\mathrm{K}}\left(\theta^{\mathrm{k}}-\Delta^{\theta} \mathrm{z}_{\mathrm{k}}\right)^{\prime}\left(\theta^{\mathrm{k}}-\Delta^{\theta} \mathrm{z}_{\mathrm{k}}\right)+\mathrm{S}\right)$

where $N=$ No of keywords, $v=10, S=10 \mathrm{I}$

$\mathrm{V}^{\gamma} \sim \mathrm{IW}\left(v+\mathrm{N}, \sum_{\mathrm{k}=1}^{\mathrm{K}}\left(\gamma^{\mathrm{k}}-\Delta^{\gamma} \mathrm{Z}_{\mathrm{k}}\right)^{\prime}\left(\gamma^{\mathrm{k}}-\Delta^{\theta} \mathrm{Z}_{\mathrm{k}}\right)+\mathrm{S}\right)$

where $\mathrm{N}=$ No of keywords, $v=10, \mathrm{~S}=10 \mathrm{I}$

$\mathrm{V}^{\alpha} \sim \operatorname{IW}\left(v+\mathrm{N}, \sum_{\mathrm{k}=1}^{\mathrm{K}}\left(\alpha^{\mathrm{k}}-\Delta^{\alpha} \mathrm{z}_{\mathrm{k}}\right)^{\prime}\left(\alpha^{\mathrm{k}}-\Delta^{\alpha} \mathrm{z}_{\mathrm{k}}\right)+\mathrm{S}\right)$

where $N=$ No of keywords, $v=10, S=10 \mathrm{I}$

Step V: Draw $\Delta^{\beta} \Delta^{\theta} \Delta^{\gamma} \Delta^{\alpha}$

Then

$\Delta^{\beta} \sim \mathrm{N}\left(\widetilde{\Delta^{\beta}}, \mathrm{q}_{\beta}\right)$

where $\mathrm{q}_{\beta}=\left[\left(\mathrm{z}_{\mathrm{k}}^{\prime} \mathrm{z}_{\mathrm{k}}\right)^{-1}+\mathrm{A}_{0}\right]^{-1} \& \widetilde{\Delta^{\beta}}=\mathrm{q}_{\beta}\left[\mathrm{z}_{\mathrm{k}}^{\prime} \beta^{\mathrm{k}}+\mathrm{A}_{0} \overline{\Delta^{\beta}}\right]$

$\overline{\Delta^{\beta}}=0, \mathrm{~A}_{0}=0.01 \mathrm{I}$

$\Delta^{\theta} \sim \mathrm{N}\left(\widetilde{\Delta^{\theta}}, \mathrm{q}_{\theta}\right)$

where $\mathrm{q}_{\theta}=\left[\left(\mathrm{z}_{\mathrm{k}}^{\prime} \mathrm{z}_{\mathrm{k}}\right)^{-1}+\mathrm{A}_{0}\right]^{-1} \& \widetilde{\Delta^{\theta}}=\mathrm{q}_{\theta}\left[\mathrm{z}_{\mathrm{k}}^{\prime} \theta^{\mathrm{k}}+\mathrm{A}_{0} \overline{\Delta^{\theta}}\right]$

$\overline{\Delta^{\theta}}=0, \mathrm{~A}_{0}=0.01 \mathrm{I}$ 


$$
\begin{array}{ll}
\Delta^{\gamma} \sim \mathrm{N}\left(\widetilde{\Delta^{\gamma}}, \mathrm{q}_{\gamma}\right) & \text { where } \mathrm{q}_{\gamma}=\left[\left(\mathrm{z}_{\mathrm{k}}^{\prime} \mathrm{z}_{\mathrm{k}}\right)^{-1}+\mathrm{A}_{0}\right]^{-1} \& \widetilde{\Delta^{\gamma}}=\mathrm{q}_{\gamma}\left[\mathrm{z}_{\mathrm{k}}^{\prime} \gamma^{\mathrm{k}}+\mathrm{A}_{0} \overline{\Delta^{\gamma}}\right] \\
\overline{\Delta^{\gamma}}=0, \mathrm{~A}_{0}=0.01 \mathrm{I} & \text { where } \mathrm{q}_{\alpha}=\left[\left(\mathrm{z}_{\mathrm{k}}^{\prime} \mathrm{z}_{\mathrm{k}}\right)^{-1}+\mathrm{A}_{0}\right]^{-1} \& \widetilde{\Delta^{\alpha}}=\mathrm{q}_{\alpha}\left[\mathrm{z}_{\mathrm{k}}^{\prime} \alpha^{\mathrm{k}}+\mathrm{A}_{0} \overline{\Delta^{\alpha}}\right] \\
\Delta^{\alpha} \sim \mathrm{N}\left(\widetilde{\Delta^{\alpha}}, \mathrm{q}_{\alpha}\right) & \\
\overline{\Delta^{\alpha}}=0, \mathrm{~A}_{0}=0.01 \mathrm{I} &
\end{array}
$$

\section{Technical Appendix A2}

In order to determine the impact of endogeneity on the parameter estimates due to strategic advertiser behavior, we conducted an experiment in which we changed the bids of ten keywords randomly on a weekly basis for a period of two months ${ }^{13}$. By randomizing the bids, we eliminate the potential endogeneity in advertiser bidding strategy. We compared the estimates obtained from this sample with those obtained from another two month sample with advertiser-selected bids for the same set of keywords. The second sample is based on the two month period preceding the experiment.

Table 5: Parameter estimates for regular and random bids

Click through rate (CTR)

\begin{tabular}{|l|l|l|}
\hline & Regular Bids & \multicolumn{1}{c|}{ Random Bids } \\
\cline { 2 - 3 } Const & $-1.24(0.1)^{\star * *}$ & $-1.19(0.03)^{\star * *}$ \\
pos & $-0.76(0.11)^{\star *}$ & $-0.63(0.02)^{\star * *}$ \\
(pos)^2 $^{*}$ & $0.06(0.03)^{\star *}$ & $0.04(0.004)^{\star * *}$ \\
\hline
\end{tabular}

Conversion rate (CVI)

\begin{tabular}{|l|l|l|}
\hline \multirow{2}{*}{ Const } & Regular Bids & \multicolumn{1}{c|}{ Random Bids } \\
\cline { 2 - 3 } pos & $-7.03(0.13)^{\star * *}$ & $-6.8(0.05)^{\star \star *}$ \\
(pos)^2 & $-0.4(0.08)^{\star \star *}$ & $1.03(0.03)^{\star * *}$ \\
\hline
\end{tabular}

We estimate the parameters for the click through rate (CTR) and conversion rate per impression (CVI) using the Hierarchical Bayesian model described in the main paper. The

\footnotetext{
${ }^{13}$ This experiment was conducted for a different advertiser that sells collectibles. The keywords in the advertiser's campaign also demonstrate a similar effect of ad position on performance as that described in the main paper. The ten keywords for the experiment were identified in consultation with the firm. We selected popular keywords for which the advertiser has to compete for position and that do not contain the firm's brand name.
} 
selected keywords have the same length. As a result, only constant term is used in the $z$ matrix and keyword specific heterogeneity is captured using the random coefficient. Comparing the estimates (Table 5) we find that the impact of position on CTR and CVI is directionally similar for both type of bids. i.e. CTR decays with position whereas CVI first increases with position and then decreases.

Using the parameter estimates, we verified that the revenue is also non- monotonic with position in both datasets. Thus although our parameter estimates change under random bidding, our results are qualitatively similar. 